\title{
Variables psicosociales asociadas a compensación metabólica de pacientes diabéticos de tipo 2
}

\author{
Anny A Q uintana ${ }^{1 a}$, José Manuel Merino ${ }^{2 b}$, \\ Pablo Merino Ric, Juan Carlos $\mathrm{Ce}^{3 \mathrm{C}}$. \\ Role of psychosocial variables in the
metabolic control of type 2 diabetics
}

Background: Patients' cultural orientations play an important role in chronic diseases. However, medical education research still does not emphasize these variables. Aim: To measure the influence of psychosocial dimensions on blood glucose control in type 2 diabetic patients. Subjects and methods: Case-control design. Data were collected from institutional records and structured interviews. Blood glucose control was tested using glycosylated hemoglobin A1C. Patients with a good metabolic profile defined as a glycosylated hemoglobin of less than $7 \%$ were considered cases while those with a glycosylated hemoglobin $\geq 7 \%$ were labeled as controls. Sixty seven cases and 61 controls were randomly selected at a public health center located in Los Angeles, Chile. Socio-demographic, illness-related, and psychosocial variables were measured and multiple modeling using logistic regression was performed. Results: Seventy percent of patients were female, mean age was 61 years, 57\% were housewives and most had a low income and educational level. The cultural predictors of metabolic control of diabetes were the perception of obstacles for blood glucose control, attribution of health benefits to a good control, family support and bonding quality with the health team. Conclusions: Cultural variables play a significant role in metabolic control of diabetic patients and must be born in mind in educational campaigns (Rev Méd Chile 2008; 136: 1007-14).

(Key words: Cultural characteristics; Diabetes mellitus, type 2; Psychology, social)

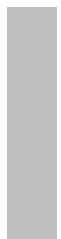

Recibido el 28 de agosto, 2007. Aceptado el 14 de abril, 2008.

${ }^{1}$ Facultad de Medicina, Universidad de Concepción, Concepción, Chile. ${ }^{2}$ Facultad de Ciencias Sociales, Universidad de Concepción, Concepción, Chile. ${ }^{3}$ Facultad de Medicina, Universidad de La Frontera, Temuco, Chile.

aNutricionista, Magister Salud Familiar

bSociólogo, $\mathrm{PhD}$.

cAlumno 6으o Medicina

In nvestigar sobre factores determinantes de la conducta relacionada con control metabólico de la diabetes mellitus exige una profunda inmersión

Correspondencia a: Dr. José Manuel Merino E. Facultad de Ciencias Sociales, Universidad de Concepción. Casilla 160C; Correo 3, Concepción, Chile. Fax: (41) 221-5860.

E mail: jmerino@udec.cl en la psicología social que permita comprender las reacciones de los pacientes una vez conocido su diagnóstico ${ }^{1,2}$. La diabetes mellitus constituye una verdadera epidemia mundial. Cifras entregadas por la International Diabetes Federation ${ }^{3}$ muestran que actualmente existen 246 millones de diabéticos y que esta cifra podría seguir aumentando con el incremento de la obesidad. En 
Latinoamérica se estima una prevalencia de 5,7\% y las proyecciones estiman 8,1\% el año $2025^{4}$.

Esta enfermedad crónica desarrolla una serie de complicaciones, que determinan un alto grado de morbilidad y mortalidad. Representa un importante número de consultas médicas, hospitalizaciones, pensiones de invalidez y muerte, que expresan un alto costo social y económico en los países.

En la comuna de Los Angeles, provincia de Bío-Bío, en marzo de 2007, se ha estimado una prevalencia de 4.253 pacientes diabéticos, con 97\% de cobertura de atención en centros de salud públicos. El impacto de intervenciones, evaluado a través de compensación de pacientes diabéticos, monitorizando niveles metabólicos a través de glicemias y hemoglobina glicosilada, en el mismo mes, alcanzaban resultados preocupantes, similares a los resultados en el resto de la provincia y el país: $24,8 \%$ de diabéticos con glicemias menores a $110 \mathrm{mg} / \mathrm{dl}$ y $31,3 \%$ de diabéticos con test de hemoglobina glicosilada menores a $7 \%{ }^{5}$.

El Ministerio de Salud desarmolla esfuerzos para un abordaje integral de las enfermedades crónicas, específicamente de la diabetes mellitus ${ }^{6}$, desde la reorientación de subprogramas de Diabetes e Hipertensión en programas más integrales como el de Riesgo Cardiovascular, hasta la reciente incorporación de esta patología al Plan de Garantías Explícitas (GES). Sin embargo, los pacientes continúan siendo atendidos principalmente sólo en aspectos biológicos y muy superficialmente en aspectos psicosociales y familiares, intervenciones que no logran modificar la conducta cotidiana de las personas diabéticas $\mathrm{y}$, por ende, el curso de su enfermedad.

Para lograr un buen control glicémico se requiere, no solamente ingesta de medicamentos, sino ajustes importantes en la alimentación habitual, control de peso y una actividad física adecuada, todos aspectos relacionados directamente con el estilo de vida. Por tanto parte de los objetivos de los planes de tratamiento debería considerar la exploración de determinantes de esa conducta, como también las variables familiares relacionadas ${ }^{7}$.

Las teorías conductuales que avalan este estudio son principalmente el Modelo de Creencias en Salud (MCS) ${ }^{8-10}$, un modelo conceptual de inspiración cognitiva, que considera que los comporta- mientos de las personas son resultado del conjunto de creencias y valoraciones internas que el sujeto desarrolla en una situación determinada; y el Modelo de Acción Razonada ${ }^{11-14}$, cuyo planteamiento central es que una conducta de salud es resultado directo de una intención comportamental; la intención depende a su vez de actitudes frente al comportamiento concreto y de valoraciones o normas subjetivas con respecto a lo apropiado de la acción comportamental.

En concordancia con esos argumentos, surgió la motivación de realizar este estudio que sustenta la hipótesis que las variables psicosociales determinan parte de la conducta de adherencia al tratamiento, influyendo en el control metabólico de los pacientes diabéticos. Se contribuye así a la identificación de factores claves que permiten articular iniciativas en el manejo de enfermedades crónicas en atención primaria.

\section{MATERIAL Y MÉTODOS}

Diseño del estudio. Estudio de casos-controles de pacientes diabéticos tipo 2 , que se controlan en el Centro de Salud 2 de Septiembre de la Ciudad de Los Angeles, Chile.

Procedimiento de muestreo. El universo estuvo constituido por 401 pacientes que se controlan en el centro de salud. Una vez aplicados criterios de exclusión, se tuvo 257 pacientes diabéticos: 132 pacientes compensados (51,3\%) y 125 pacientes descompensados $(48,7 \%)$

Se excluyeron del estudio pacientes diabéticos mayores de 75 años, con domicilio rural, con menos de un año en tratamiento y sin examen actualizado de hemoglobina glicosilada.

Para el estudio se seleccionó aleatoriamente una muestra representativa, aplicando los porcentajes observados de control glicémico: $51,3 \%$ de pacientes compensados (67 casos) y $48,7 \%$ de pacientes descompensados (61 controles).

\section{Variables principales}

La variable principal es el control metabólico del paciente, considerándose como casos los pacientes compensados [test actual de hemoglobina glicosilada $\left(\mathrm{HbA}_{1 \mathrm{c}}\right)$ menor a $7 \%$ y pacientes 
descompensados -controles- (niveles de $\mathrm{HbA}_{1 \mathrm{c}}$ iguales o mayores a 7\%). Fueron además consideradas en el instrumento las siguientes dimensiones:

Variables demográficas: edad, sexo, escolaridad, ingreso mensual y ocupación.

Variables propias de la enfermedad: tiempo de diagnóstico, tipo de tratamiento, estado nutricional.

Variables psicosociales: basadas en el Modelo de Creencias en Salud ${ }^{8}$, se utilizó un conjunto de preguntas en formato Likert ${ }^{15}$, orientadas a medir:

- susceptibilidad percibida: es la valoración subjetiva del paciente acerca de cómo la enfermedad afectará su salud. Hay pacientes que no creen en que la diabetes les provocará serios daños; mientras otros creen que la enfermedad requiere ser enfrentada con apoyo médico constante.

- severidad percibida: los pacientes valoran los efectos que la enfermedad puede ocasionarles si no la controlan: pérdida de tiempo de trabajo, malestares físicos progresivos, dificultades sociales, invalidez, susceptibilidad progresiva frente a patologías futuras.

- beneficios atribuidos al tratamiento médico: enfrentamiento de la enfermedad en términos de adhesión a los tratamientos e indicaciones médicas es lo esperado de pacientes que han aceptado su susceptibilidad y reconocido la severidad de la diabetes. La adhesión al tratamiento es, en parte, función de esta creencia.

- barreras para enfrentar la enfermedad: la adhesión no puede tener lugar cuando a pesar de reconocer los beneficios del tratamiento médico, no se pueden superar los obstáculos que se enfrentan para su ejecución. Estas barreras pueden alejar a la persona del cumplimiento deseado.

La recolección de datos se realizó mediante cuestionario para variables sociodemográficas y variables propias de la enfermedad, con excepción del estado nutricional que se obtuvo de registros institucionales. El cuestionario fue aplicado en visitas domiciliarias a cada paciente del estudio, previo consentimiento informado para participar en la investigación.
Estadísticas. Los datos fueron procesados descriptivamente mediante Statistical Package for the Social Sciences (SPSS). El programa Statistical Analysis System (SAS), fue utilizado para la modelización multivariada de los datos primarios provenientes del cuestionario y de los datos secundarios extraídos desde los registros institucionales.

Todas las variables fueron comparadas en distribuciones univariadas, bivariadas y multivariadas para el total de los pacientes y principalmente al interior de los casos (pacientes compensados) y de los controles (pacientes descompensados). Los resultados obtenidos se exponen en la siguiente sección.

\section{RESULTADOS}

El perfil de las muestras de pacientes diabéticos tipo 2 del Cesfam 2 de Septiembre de Los Angeles indica que la mayor parte (70\%) pertenecía al sexo femenino, eran mayores de 60 años (62\%), la mayoría con educación básica incompleta (67\%), disponían de un ingreso familiar mensual inferior a 150 mil pesos (75\%), se desempeñaban principalmente como dueñas de casa o desarrollaban ocupaciones de bajo nivel de calificación (75\%). Toda esta información socio-demográfica indica que el paciente diabético tipo 2, controlado en el servicio de salud público de Los Angeles, es una persona de bajo nivel de estatus socioeconómico, ocupacional y educacional (Tabla 1).

Características de la enfermedad. Los pacientes diabéticos de tipo 2 seleccionados tenían un promedio de casi 7 años de enfermo crónico. Casi 60\% tenía menos de cinco años desde que le fue diagnosticada la enfermedad, 20\% tenía una diabetes entre 6 y 10 años de antigüedad, mientras 18\% eran diabéticos de más de 11 años. En cuanto al tipo de tratamiento que declararon realizar los pacientes, sólo $18 \%$ realizaba simultáneamente dieta, tratamiento medicamentoso y actividad física dirigida, lo que puede considerarse un tratamiento médico integral de la enfermedad. Un poco más de un tercio (36,7\%), reconoció sólo seguir el tratamiento medicamentoso, excluyendo la dieta supervisada y la actividad física sistemática de su tratamiento habitual. El 44,5\% de los pacientes, acompañaba el 
Tabla 1. C aracterísticas sociodemográficas pacientes diabéticos tipo II. C ESFAM 2 de septiembre, comuna Los Ángeles, año 2007

\begin{tabular}{|c|c|c|}
\hline & $\mathrm{n}$ & $\%$ \\
\hline \multicolumn{3}{|l|}{ Sexo } \\
\hline Hombres & 38 & 29,7 \\
\hline Mujeres & 90 & 70,3 \\
\hline Total & 128 & 100,0 \\
\hline \multicolumn{3}{|l|}{ Edad } \\
\hline $30-39$ & 5 & 3,9 \\
\hline $40-49$ & 20 & 15,6 \\
\hline $50-59$ & 23 & 18,0 \\
\hline $60-69$ & 48 & 37,5 \\
\hline 70 y más & 32 & 25,0 \\
\hline Total & 128 & 100,0 \\
\hline \multicolumn{3}{|l|}{ Nivel Educacional } \\
\hline Sin Educación & 12 & 9,4 \\
\hline Básico & 74 & 58,3 \\
\hline Media & 39 & 30,7 \\
\hline Superior & 2 & 1,6 \\
\hline Total & 127 & 100,0 \\
\hline \multicolumn{3}{|l|}{ Nivel socioeconómico } \\
\hline$<\$ 100.000$ & 68 & 53,1 \\
\hline$\$ 100.000-150.000$ & 28 & 21,9 \\
\hline$\$ 150.000-200.000$ & 17 & 13,3 \\
\hline$>\$ 200.000$ & 15 & 11,7 \\
\hline Total & 128 & 100,0 \\
\hline \multicolumn{3}{|l|}{ Ocupación } \\
\hline Dueña de casa & 73 & 57,0 \\
\hline Obreros, manipuladores, auxiliares & 17 & 13,3 \\
\hline Maestros, carpinteros & 3 & 2,3 \\
\hline Comerciantes & 4 & 3,1 \\
\hline Pensionados, jubilados & 29 & 22,7 \\
\hline Secretarias, técnico paramédico & 2 & 1,6 \\
\hline Total & 128 & 100,0 \\
\hline
\end{tabular}

tratamiento medicamentoso con la dieta indicada por el nutricionista, pero excluía de su tratamiento la actividad física planificada. Resulta sorprendente que $80 \%$ de los diabéticos de tipo 2 de este estudio no considera el ejercicio físico como parte importante del tratamiento integral de su enfermedad.

En relación al estado nutricional del paciente diabético, evaluado por el índice de masa corporal (IMC), sólo 7\% tenía un índice normal (IMC inferior a $24,9 \mathrm{~kg} / \mathrm{m}^{2}$ ). Es sorprendente que pese a ser pacientes regularmente controlados por un servicio público, $63 \%$ tenía un IMC superior a 30 $\mathrm{kg} / \mathrm{m}^{2}$, lo que lo clasifica como obeso. Otro $30 \%$ de los pacientes fue clasificado como con sobrepeso porque su medición está entre 25 y $29,9 \mathrm{~kg} /$ $\mathrm{m}^{2}$. Es necesario subrayar que $93 \%$ de estos pacientes diabéticos controlados en el servicio estudiado tenía valores de IMC que lo clasifican como personas con sobrepeso o en estado de obesidad manifiesta (Tabla 2). 
Tabla 2. C aracterísticas de la enfermedad pacientes diabéticos tipo 2. CESFAM 2 de septiembre, comuna Los Ángeles, año 2007

\begin{tabular}{|lrr|}
\hline HB A1C & $\mathrm{n}$ & $\%$ \\
\hline$\geq 7,0$ (control) & 61 & 47,7 \\
$<7,0$ (caso) & 67 & 52,3 \\
Total & 128 & 100,0 \\
Tiempo diagnóstico (años) & & \\
$1-5$ & 76 & 60,8 \\
$6-10$ & 26 & 20,6 \\
$11-20$ & 19 & 15,2 \\
$21-30$ & 4 & 2,3 \\
Total & 125 & 100,0 \\
Tipo de tratamiento & & \\
Dieta + Actividad física & 1 & 0,8 \\
Sólo medicamentos & 47 & 36,7 \\
Dieta + medicamentos & 57 & 44,5 \\
Dieta + Actividad física +medicamentos & 23 & 18,0 \\
Total & 128 & 100,0 \\
Estado nutricional (IMC) & & \\
18,5-24,9 (normal) & 9 & 7,0 \\
25,0-29,9 (sobrepeso) & 38 & 29,7 \\
$\geq 30$ (obeso) & 81 & 63,3 \\
Total & 128 & 100,0 \\
\hline
\end{tabular}

Modelización multivariada del control metabólico. Se exponen ahora los procedimientos empleados para identificar estadísticamente los factores psicosociales que influyen en la compensación diabética de tipo 2. Para tal propósito se ha desarrollado una modelización multivariada mediante regresión logística ${ }^{16,17}$ para lo que se ha modelado la compensación diabética (casos $=1$ ) como el resultado de interés analítico.

En la Tabla 3 se presentan los resultados de la modelización del control metabólico. El modelo nulo identifica la variación total del control metabólico: el valor del estadístico coeficiente de verosimilitud $^{18,19}$ (-2 Log L) es igual a 117,2, lo que debe ser interpretado como la cantidad máxima de variación a ser explicada por los predictores en la modelización ${ }^{20}$. Los modelos bivariados indican que se encontró cuatro predictores psicosociales con influencia individual significativa sobre la compensación de los pacientes diabéticos: beneficios atribuidos por el paciente al tratamiento médico que lo conduce al control metabólico; barreras percibidas por el paciente que obstaculizan ese tratamiento; relación del paciente con el equipo de salud y, por último, disponibilidad declarada por el paciente de apoyo familiar para la ejecución del tratamiento. Entre las cuatro orientaciones culturales anteriores, la más importante para la compensación del diabético tipo 2 resultó ser la atribución de beneficios que el paciente efectúa respecto del tratamiento médico que conduce al control metabólico. Esta variable sola reduce 36,7 puntos respecto de la variación total $(177,2)$, esto es, $20 \%$ de ese total, lo que permite concluir que es la variable psicosocial con mayor influencia sobre el control metabólico (Tabla 3).

En el segundo paso de la modelización, se estimó una ecuación de regresión logística múltiple con todos los predictores significativos detectados. En esta ecuación que simultáneamente incluye los cuatro predictores, se explican 60,3 puntos de los 177,2 totales. Esto indica que las cuatro orientaciones culturales explican un tercio 
Tabla 3. M odelización de regresión logística multivariada de control metabólico

\begin{tabular}{|c|c|c|c|c|c|}
\hline Variable & $-2 \log \mathrm{L}$ & DF & Desviación & $\mathrm{X} 2$ & $\mathrm{P}$ \\
\hline \multicolumn{6}{|l|}{ Modelo Nulo } \\
\hline Control metabólico & 177,164 & & & & \\
\hline \multicolumn{6}{|l|}{ Modelos bivariados } \\
\hline Conocimiento & & 1 & 174,890 & 2,2747 & 0,1315 \\
\hline Creencias & & 1 & 176,294 & 0,8700 & 0,3509 \\
\hline Beneficios & & 1 & 140,495 & 36,669 & $<0,0001$ \\
\hline Barreras & & 1 & 140,705 & 36,458 & $<0,0001$ \\
\hline Relación con equipo de salud & & 1 & 152,002 & 25,163 & $<0,0001$ \\
\hline Apoyo familiar & & 1 & 142,329 & 34,835 & $<0,0001$ \\
\hline \multicolumn{6}{|l|}{ Modelo 4 predictores significativos } \\
\hline Beneficios + barreras + apoyo + relación equipo & & 4 & 116,897 & 60,267 & $<0,0001$ \\
\hline Beneficios & & 1 & & 6,4999 & 0,0108 \\
\hline Barreras & & 1 & & 9,5525 & 0,0020 \\
\hline Apoyo familiar & & 1 & & 2,4443 & 0,1179 \\
\hline Relación equipos de salud & & 1 & & 1,2400 & 0,2655 \\
\hline
\end{tabular}

del total de variación de compensación diabética. Ciertamente los tres predictores que se agregaron al beneficio atribuido por el paciente al tratamiento elevan la explicación en $14 \%$, lo que es un avance en la identificación de predictores psicosociales del control metabólico. Sin embargo, el examen detenido de la última ecuación de regresión logística, indica que en el modelo óptimo (número final de variables con efectos sobre la compensación diabética) sólo los beneficios atribuidos al tratamiento y la percepción de barreras que se oponen a ese tratamiento, mantienen efectos directos significativos sobre el control metabólico, constituyéndose así en las variables culturales más importantes del modelo final. Un análisis detallado del significado de las orientaciones culturales que se asociaron significativamente al control metabólico de la diabetes tipo 2, indica que los pacientes que atribuyen más beneficios al tratamiento prescrito por el médico como condición indispensable para sentirse mejor y valoran mejor sus indicaciones como modificaciones positivas de estilos de vida, son quienes tienen mejor control metabólico. Las barreras percibidas por el paciente para el cumplimiento del tratamiento médico están referidas a las cosas que se interponen para cumplir adecuadamente esta prescripción. Los pacientes indican que el tratamiento es demasiado problemático para seguirlo, puesto que los obliga a hacer modificaciones en su conducta muy sacrificadas y dolorosas, o incluir en la dieta modificaciones de muy alto costo.

\section{DisCUSIÓN Y CONCLUSIONES}

De los cuatro indicadores psicosociales que tienen efectos individuales sobre el control metabólico, en el modelo final mantienen efectos directos la atribución de beneficios y la percepción de barreras, por lo que deben considerarse como variables con efectos robustos sobre la compensación que conduce al control metabólico de la diabetes tipo 2. Mientras más beneficios se atribuye por el paciente al tratamiento que asegura ese control y mientras menos barreras se perciben para asegurar la ejecución del tratamiento, mayor es la probabilidad de que el paciente esté compensado metabólicamente. El apoyo familiar disponible y las relaciones positivas con el equipo de salud actúan indirectamente en ese modelo final.

Se acepta la hipótesis postulada que señala la existencia de relaciones entre variables psicosociales y control metabólico de la diabetes tipo 2 . Específicamente se encontraron vínculos directos entre las creencias que los enfermos tienen res- 
pecto a los beneficios y barreras percibidas respecto del plan de cuidado, correspondiendo a estas dos dimensiones, la mayor significación estadística respecto al control metabólico en la muestra explorada. En consecuencia, se ha encontrado plena concordancia en nuestra hipótesis respecto de la dirección de las teorías psicológicas contemporáneas de determinantes de la conducta en que se postulan actitudes, creencias e intenciones como los factores influyentes más próxi$\operatorname{mos}^{21,22}$. Se ha ratificado en este estudio el hecho de que las creencias, así como otras orientaciones culturales de los pacientes, constituyen un área de enorme importancia en los fenómenos asociados a salud ${ }^{23,24}$. Particularmente se ha demostrado la influencia directa que ejerce sobre el control metabólico de la diabetes la creencia del paciente acerca de los beneficios atribuidos al tratamiento médico, en forma simultánea con sus creencias acerca de las barreras que puede obstaculizar ese tratamiento. Las relaciones positivas con el equipo de salud mantienen su influencia indirecta porque son ellos quienes deben orientar y reforzar la decisión del paciente en orden a que los esfuerzos para cumplir el tratamiento son indispensables, para evitar complicaciones y patologías mayores. $\mathrm{El}$ rol indirecto de las redes familiares radica en su

\section{REFERENCIAS}

1. LóPEZ G. Nueva clasificación y criterios diagnósticos de la diabetes mellitus. Rev Méd Chile 1998; 126: 833-7.

2. Garza M, Calderón C, Salinas M, Núñez G, Viliarroel E. Atribuciones y creencias sobre la Diabetes Mellitus tipo 2. Rev Méd México 2003; 41: 465-72.

3. International Diabetes Federation (IDF), Diabetes Atlas, $3^{\text {rd }}$ Edition, Brussels, Belgium, 2006.

4. Organización Mundial de la Salud (OMS), Informe Macroeconomía y salud. Geneve, 2000.

5. Servicio Salud Bío Bío (SSBB), Monitoreos Control Metabólico Pacientes Diabéticos Bajo Control Establecimientos APS, Los Angeles, 2007.

6. Ministerio de Salud - Chile. Guía Clínica Diabetes Mellitus Tipo 2. Serie Guías Clínicas MINSAL, 1a Edición, Santiago, 2006.

7. Rowand J. Familias, enfermedad y discapacidad. Una propuesta desde la terapia sistémica. España: Editorial Gedisa, 2000.

8. BECKER MH (ED). The health belief model and perso- capacidad de movilizar recursos para aumentar las fortalezas personales dirigidas al enfrentamiento positivo de dificultades o barreras percibidas en la enfermedad.

El control metabólico, en cuanto aspecto predominantemente biológico de una enfermedad como la diabetes mellitus tipo 2, es ponderable principalmente en términos de glicemias y exámenes de hemoglobina, pero tiene también un componente fuertemente cultural constituido por un conjunto de orientaciones subjetivas que se entrelazan con lo médico para conformar los resultados de salud. Este estudio es una ilustración de cómo valorar las contribuciones de esos factores subjetivos para diseñar estrategias y tratamientos más integrales. Dicha contribución implica considerar en los planes de cuidado para pacientes diabéticos aspectos culturales que se relacionan con las conductas en salud, tales como sus creencias relacionadas con la susceptibilidad frente a la diabetes, la severidad asociada a la enfermedad, los beneficios atribuidos al tratamiento médico y los principales obstáculos para adherir que los pacientes perciben, porque son los elementos que actuarán sistemáticamente como facilitadores o gatilladores de las modificaciones indispensables en las conductas que obstaculizan la salud.

nal health behaviors. Thorofare, NJ: Charles B. Slack, Inc., 1974.

9. Harrison JA, Mulen PD, Green LW. A metaanalysis of studies of the health belief model. Health Education Research 1992; 7: 107-16.

10. Moreno E, Rosales J. El modelo de creencias en salud: Revisión teórica, consideración crítica y propuesta alternativa. I: Hacia un análisis funcional de las creencias en salud. Internacional Journal of Psychology and Psychological Therapy 2003; 3: 91-109.

11. AzJEN I. The theory of planned behavior. Organizational Behavior and Human Decision Process 1991; 50: 179-211.

12. AzJen I, Fishbein M. Understanding attitudes and predicting behaviors. Englewoods Cliffs, NJ: Prentice Hall, 1980.

13. Carpi A, Breva A, Palmero F. La teoría de la acción planeada y la reducción del estrés percibido para prevenir la enfermedad cardiovascular. Anales de Psicología 2005, 21: 84-91.

14. Gretebeck KA, Black DR, Blue CL, Glickman LT, Huston SA, Gretebeck RT. Physical activity and 
function in older adults: Theory of planned behavior. Am J Health Behav. 2007; 31: 203-14.

15. Streiner D, Norman G. Health measurement scales. New York: Oxford University Press, 1995.

16. MERINO JM. Modelización multivariada de variables dependientes categóricas: Una introducción a la regresión logística. Ciencia y Enfermería 1996; II: 27-40.

17. Merino JM, Olavarría S, Isla X. Efectos-vecindario sobre la salud reproductiva de la mujer chilena. Rev Méd Chile 2003; 131: 987-95.

18. Silva LC. Excursión a la regresión logística en ciencias de la salud. Madrid: Ediciones Díaz de Santos, SA. 1995.

19. Cowet D. Modelling binary data. London: Chapman Hall, 1991.

20. AgRESTI A. An introduction to categorical data analysis. New York: John Wiley \& Sons, 1996.
21. Perkins MB, Jensen PS, Jaccard J, Golwitzer $P$, Oettingen G, Pappadopulos E, Hoagwood KE. Applying theory-driven approaches to understanding and modifying clinician's behaviors: What do we know? Psychiatric Services 2007; 58: 342-8.

22. HaRdeman W, Johnston M, Johnston D, Bonnetti D, WAREHAM NJ, KinMONTH AL Application of the theory of planned behavior in change interventions: A systematic review. Psychology and Health 2002; (17): 123-58.

23. Godin G, Ick G. The theory of planned behavior: A review of its applications to health-related behaviors. Am J Health Prom 1996; 2: 87-98.

24. Baban A, CRACIUN C. Changing health-risk behaviors: A review of theory and evidence-based interventions in health psychology. Journal of Cognitive and Behavioral Psychotherapies. 2007; 7: 45-67. 